\title{
Decreased BOLD responses in audiovisual processing
}

Esther Wiersinga-Post ${ }^{\mathrm{a}, \mathrm{b}, \mathrm{c}}$, Sonja Tomaskovic ${ }^{\mathrm{a}, \mathrm{d}}$, Lavinia Slabu ${ }^{\mathrm{a}, \mathrm{e}}$, Remco Renken ${ }^{\mathrm{a}}$, Femke de Smit ${ }^{\mathrm{a}}$ and Hendrikus Duifhuis ${ }^{\mathrm{a}}$

a Neuroimaging Center, Graduate School for Behavioural and Cognitive Neurosciences, University of Groningen, The Netherlands,

b Department of Artificial Intelligence, University of Groningen, The Netherlands,

c Department of Otorhinolaryngology, University Medical Center Groningen, The Netherlands,

d Neurosciences Research Institute, School of Life and Health Sciences, Aston University, UK and

e Cognitive Neuroscience Research Group, Department of Psychiatry and Clinical Psychobiology, University of Barcelona, Spain

Correspondence: Esther Wiersinga-Post, PhD, University Medical Center Groningen, Hanzeplein 1, Groningen $9713 \mathrm{AV}$, The Netherlands

Tel: + 3150 3611337; fax: + 3150 3611698; e-mail: j.e.c.wiersinga-post@med.umcg.nl

Keywords: audiovisual binding, audiovisual integration, crossmodal, deactivation, fMRI, human brain imaging, inhibition, McGurk effect, multimodal, sparse sampling 


\begin{abstract}
Audiovisual processing was studied in a functional magnetic resonance imaging study using the McGurk effect. Perceptual responses and the brain activity patterns were measured as a function of audiovisual delay.

In several cortical and subcortical brain areas, BOLD responses correlated negatively with the perception of the McGurk effect. No brain areas with positively correlated BOLD responses were found. This was unexpected as most studies of audiovisual integration use additivity and super additivity - that is, increased BOLD responses after audiovisual stimulation compared with auditoryonly and visual-only stimulation - as criteria for audiovisual integration. We argue that brain areas that show decreased BOLD responses that correlate with an integrated audiovisual percept should not be neglected from consideration as possibly involved in audiovisual
\end{abstract}

\title{
Introduction
}

To perceive objects and events in the environment, organisms are equipped with different senses each specialized for the detection of certain physical aspects of the outside world. How the brain manages to combine information that enters through separate sensory chan- nels and forms a unified percept of the outside world is still not well understood.

This study investigates which brain areas are involved in audiovisual (AV) binding using functional magnetic resonance imaging (fMRI) combined with behavioral measurements of the McGurk effect [1]. This effect is very suitable for studying AV binding, as the auditory percept of McGurk stimuli depends on whether AV information is integrated or processed separately. Stimuli that easily elicited the McGurk effect can for instance be made by dubbing the syllables /aba/ and /apa/ on movies of a mouth pronouncing the non-labials /aga/ and /aka/. As the consonants $/ \mathrm{b} / \mathrm{and} / \mathrm{p} /$ are easily confused with the consonants $/ \mathrm{d} /$ and $/ \mathrm{t} /$ respectively [2], and because these are visually very similar to the visemes /g/ and / $/$ / [3], individuals sensitive to the McGurk effect will perceive /ada/ and /ata/ when listening to these incongruent stimuli [1]. Accordingly, the perception of /ada/ or /ata/ indicates that auditory and visual information is fused, whereas the perception of /aba/ or /apa/ indicates that auditory and vsual information is processed separately.

In our experiment, the synchronization of the McGurk stimuli was varied to manipulate the strength of the McGurk effect (i.e. the strength of AV binding). We hypothesize that the brain areas involved in the AV binding process will show brain activation patterns correlated to the strength of the McGurk effect. Brain activation patterns as measured using the BOLD response, were therefore correlated to the strength of the AV binding as indicated by the perceptual responses.

To use a McGurk response as evidence for the fusion of auditory and visual information, it is important that the auditory stimulus is easily discerned. If not, the 'changed' auditory percept could simply be caused by the absence of auditory information. To ensure the audibility of the relatively brief and weak consonants in the speech stimuli, we used a sparse sampling paradigm [4,5]. Stimuli in this paradigm are presented in periods of rela- tive silence between scanning periods. This ensures that the auditory stimuli are not masked by scanning noise and that the brain activation obtained depends on the stimulus and not on the scanning noise.

\section{Methods Participants}

Twenty healthy volunteers with normal hearing and normal or corrected-to-normal vision participated in this study. None of them reported neurological or language impairments. Four (two females) out of the initial pool of 20 individuals were excluded from the fMRI analysis because of the absence of the McGurk effect as revealed in the psychophysical study. One individual did not complete the scanning experiment and one individual was excluded because of excessive motion during 
scanning. We analyzed a total of 14 individuals aged between 22 and 45 years (five females). Before participa- tion, individuals signed an informed consent form. The experiment was approved by the local Medical Ethical Committee, Groningen.

\section{Stimuli}

Movies were made with a digital video camera [Panasonic 3 CCD 24 digital zoom (Panasonic, One Panasonic Way, Secausus, NJ07094, USA)] and external microphone (Sennheiser) in which a female Dutch speaker uttered the syllables /aba/, /aga/, /apa/ and /aka/. The video frame rate was $25 \mathrm{~Hz}$ and the sounds were digitized at a sampling rate of $48 \mathrm{kHz}$ with 16-bit resolution. The movies were edited in Adobe Premiere (Adobe Premiere, 345 Park Avenue, San Jose, California, 95110-2704 USA). Incongruent stimuli were produced in which the audio signals of the /aba/ and /apa/ utterances were dubbed onto the videos of the speaker saying /aga/ and /aka/, respect- ively. Stimuli with AV delays ranging from $-510 \mathrm{~ms}$ (audio leads video) to $+420 \mathrm{~ms}$ (video leads audio) were prepared. The duration of the acoustic syllables was around $730 \mathrm{~ms}$. The total length of the AV movies was $2.00 \mathrm{~s}$ with the auditory utterance temporally centred. Only the mouth was shown. To avoid onset and offset information because of the opening and closing of the mouth, the movies started and ended with an open mouth.

The psychophysical experiment used 14 AV stimuli with different delays $(-510,-390,-270,-$ $190,-110,-70,-30,+10,+50,+90,+130,+210,+290$ and $+410 \mathrm{~ms})$. AV stimuli were selected for the fMRI experi- ment that resulted in minimal $(-510$ and $+410 \mathrm{~ms})$, in- termediate $(-110$ and +210 $\mathrm{ms}$ ) and maximal (+ $50 \mathrm{~ms}$ ) strength McGurk effects. Furthermore, audio-only (A) stimuli (speech sounds /aba/ or /apa/ combined with a blank screen with a fixation cross), and visual-only (V) stimuli (a mouth pronouncing /aga/ or /aka/) were used. The baseline stimulus was a fixation cross on the screen.

Stimuli were presented in the psychophysical and the fMRI experiments against a continuously present pink noise background of $40 \mathrm{~dB}$ SPL. Along with the use of ear mufflers, which reduced the scanner noise by approxi- mately $20 \mathrm{~dB}$, this noise background was high enough to mask environmental noise during the relatively silent periods between the acquisitions. The signal-to-noise ratio of the auditory stimuli was $20 \mathrm{~dB}$.

\section{Psychophysical experiment}

Individuals were seated in a sound insulated booth (Industrial Acoustic Company, Sohlweg 17, D-71372 Niederkrchten, Deutschland). Auditory stimuli were presented through headphones [Telephonics TDH-49P (Telephonics, 815 Broad Hollow Road, Farmingdale, New York 11735, USA)] and visual stimuli were pre- sented on a computer screen at $60 \mathrm{~cm}$ distance. The two AV stimuli with 14 different AV delays were presented seven times in pseudo-random order, resulting in a total of 196 stimulus presentations per session. Individuals had to indicate whether they heard the sound /aba/, /ada/, / apa/ or /ata/ by pressing corresponding keys on a keyboard. Each individual performed three sessions.

\section{Functional magnetic resonance imaging experiment}

Functional MR scans were acquired using a $3 \mathrm{~T}$ scanner (Philips Intera, Philips Medical Systems, Eindhoven, The Netherlands). Prior to the functional images, a T1- weighted anatomical scan of the entire brain was produced (160 slices; field of view $=256 \mathrm{~mm}$; scanning matrix $=256256$; transverse slice orientation; repeti- tion time $=25 \mathrm{~ms}$; echo time $=4.6 \mathrm{~ms}$; flip angle $=301)$. Functional MR images were acquired using echo planar imaging; (46 slices; slice thickness $=3.5 \mathrm{~mm}$; field of view $=224 \mathrm{~mm}$; scanning matrix $=64$ 64; transverse slice orientation; repetition time $=20 \mathrm{~s}$; echo time $=35 \mathrm{~ms}$; flip angle =901). Eight different stimulus conditions were used: 1 to 5 - AV with delays of $510,-110,50,210$ and $410 \mathrm{~ms} ; 6-\mathrm{A} ; 7-\mathrm{V}$; and $8-$ baseline. Each experiment consisted of two functional series in which 74 volumes were scanned (18 volumes at baseline and 56 volumes where the 
seven remaining stimulus conditions were presented eight times).

To avoid interference with scanner noise during acoustic stimulus presentation, we applied the sparse sampling method [4,5] with a relatively long repetition time of $20 \mathrm{~s}$. Between two acquisitions, four stimuli of one stimulus condition were presented in pseudo-random order.

The entire experiment, including one anatomical scan and two functional series, lasted approximately $50 \mathrm{~min}$. After each stimulus presentation, except after a baseline stimulus, individuals had to indicate what was said (/aba/, /ada/, /apa/ or /ata/) by pressing a button on a response box with their left or right index fingers, or their middle finger. The individuals had to respond within $1 \mathrm{~s}$. During this second, a window with four buttons corresponding to the possible answers was displayed.

Visual stimuli were projected onto a screen located at the base of the scanner through a LCD projector (Barco G300j, President Kennedypark 35, 8500 Kortrijk, Belgie). Individuals viewed the movies through a mirror angled above the head. Auditory stimuli were presented through the headphones of the scanner's sound system.

\section{Data analysis}

The SPM2 software (http://www.fil.ion.ucl.ac.uk/spm) was used to process and analyze the data. The echo planar imaging images were realigned to the first volume in the run. These images were co-registered with the individuals' own anatomical images. They were normalized to the standard brain (MNI) and spatially smoothed using a Gaussian kernel with a full width half maximum of $10 \mathrm{~mm}$.

For each individual, weighted effect sizes (con values) were obtained for all stimulus conditions (1-7) against baseline (8). A block design was used for this analysis, without convolution with the hemodynamic response function, as sparse sampling was used. To correlate the individuals' McGurk responses with the brain activation levels, a second level analysis was applied. The con values for conditions 1-5 were correlated to the average McGurk responses across individuals (the regressor is shown in the last graph of Fig. 1, black symbols). We also analyzed the data using the individual mean McGurk responses as regressors (see Fig. 1, 1-14, black symbols). To the resulting t-map, a threshold of $\mathrm{P}=0.001$ (cluster size $\mathrm{Z} 20$ ) was applied. For these regions, the con values per individual were extracted using the MARSBAR toolbox (http://marsbar. sourceforge.net). 


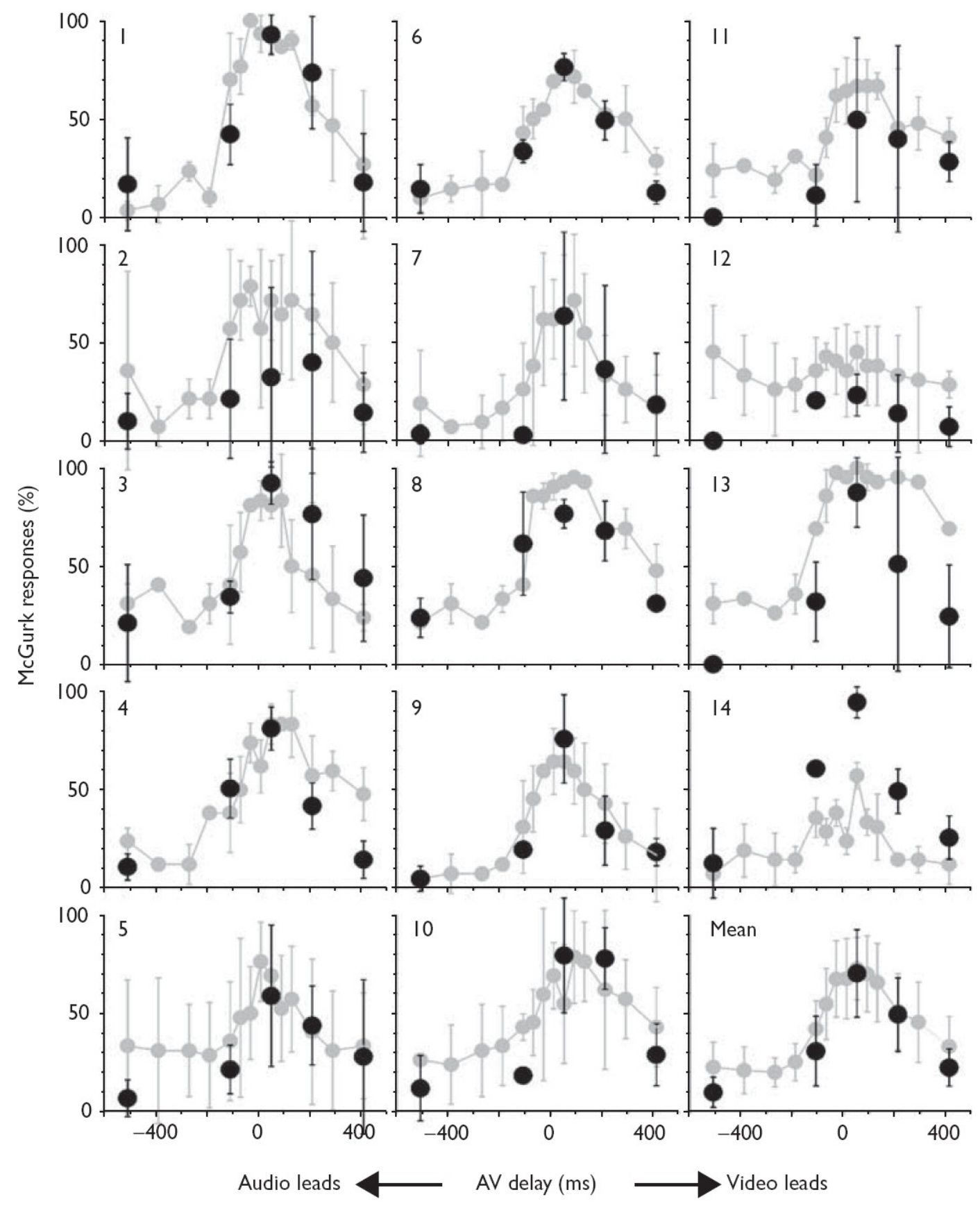

Figure. 1 Behavioral results for the 14 individual participants that were included in the fMRI experiment. Mean results for all individuals with standard deviations are shown in the last graph. Audiovisual (AV) delay in the synchronization of the AV stimuli is shown on the horizontal axis. The vertical axis represents the percentage of McGurk responses, that is, the mean with standard deviation of the /ada/ and /ata/ responses. Grey symbols represent the psychophysical experiment data. Black symbols represent the data recorded during the fMRI experiment. 


\section{Results \\ Psychophysics}

The mean percentage of McGurk responses - that is the mean of the /ada/ responses following auditory /aba/ stimulation and the /ata/ responses after auditory /apa/ stimulation - clearly depends on AV synchronization (Fig. 1). The McGurk response percentages peak in the AV delay range of -30 to $+130 \mathrm{~ms}$. Four out of the initial pool of 20 individuals always heard /aba/ or /apa/, independently of the synchronization of the AV stimulus and were excluded from the fMRI experiment (data not shown in Fig. 1).

\section{Functional magnetic resonance imaging Psychophysics}

We analyzed the whole brain and selected those brain areas in which the BOLD response showed significant correlation with the McGurk response percentages (last graph of Fig. 1, black symbols). Positive correlations were not found. Negatively correlated BOLD responses were found in the supramarginal gyrus, the superior frontal gyrus, parts of the cerebellum and the thalamus (see Table 1 and Fig. 2). All these brain areas show decreased con values after synchronous AV stimulation (see stimulus 3 in Fig. 2b) compared to the maximum response after monosensory stimulation (see stimulus 6 or 7 in Fig. 2b). All con values, except for those in the thalamus, are however positive compared to the rest condition. We also analyzed the fMRI-data using the individual average McGurk response as a regressor (Fig. 1, 1-14, black symbols). The results of this analysis are comparable with the results described (not shown).

Table 1. Brain areas in which the activation showed a significant negative correlation with the number of McGurk responses $(P=0.001$, uncorrected, cluster size $\mathrm{Z} 20)$

\begin{tabular}{|c|c|c|c|c|c|c|c|c|}
\hline & & \multicolumn{4}{|c|}{ MNI coordinates } & \multirow[b]{2}{*}{ Cluster size } & \multirow[b]{2}{*}{$t$ statistics } & \multirow[b]{2}{*}{$P$ value } \\
\hline & & Lateralization & $x$ & $y$ & $z$ & & & \\
\hline 1 & Supra marginal gyrus & $\mathrm{R}$ & 64 & -40 & 36 & 63 & 4.16 & $<0.000$ \\
\hline 2 & Cerebellum vermis & $\mathrm{R}$ & 12 & -48 & -24 & 25 & 4.16 & $<0.000$ \\
\hline 3 & Cerebellum vermis & $\mathrm{L}$ & -12 & -44 & -26 & 93 & 4.01 & $<0.000$ \\
\hline 4 & Superior frontal gyrus & L & -14 & 18 & 60 & 67 & 3.70 & $<0.000$ \\
\hline 5 & Thalamus & $\mathrm{R}$ & 6 & -10 & 2 & 20 & 3.82 & $<0.000$ \\
\hline 6 & Cerebellum & $\mathrm{L}$ & -24 & -80 & -18 & 281 & 3.71 & $<0.000$ \\
\hline 7 & Cerebellum & $\mathrm{R}$ & 32 & -72 & -20 & 69 & 3.60 & $<0.0005$ \\
\hline
\end{tabular}


(a)

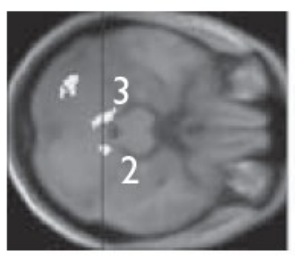

$Z=-24 \mathrm{~mm}$

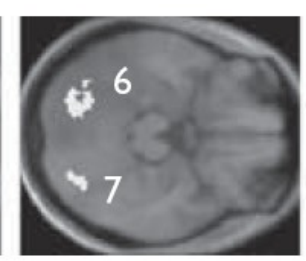

$Z=-20 \mathrm{~mm}$

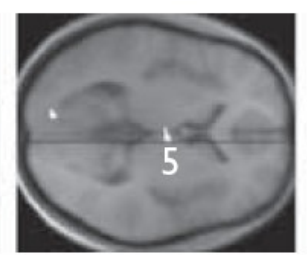

$Z=2 \mathrm{~mm}$

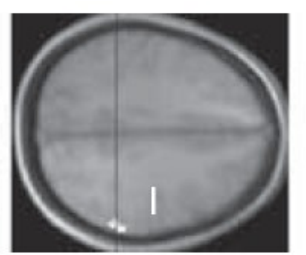

$Z=36 \mathrm{~mm}$

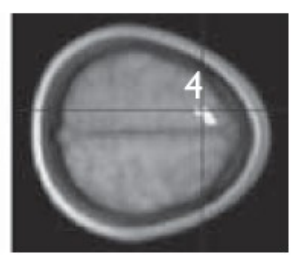

$Z=60 \mathrm{~mm}$

(b)

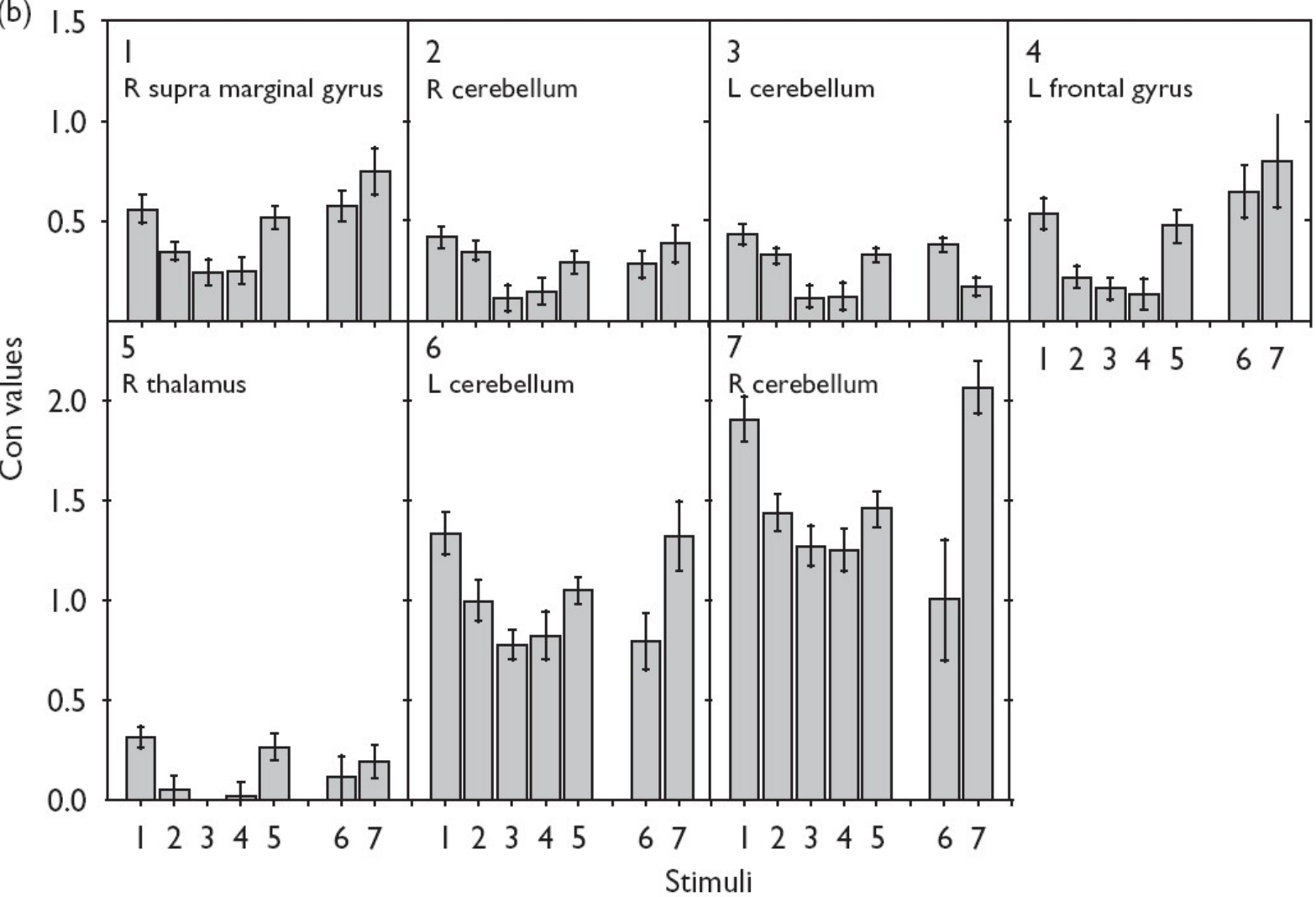

Figure 2. (a) Brain areas in which the BOLD response showed a significant negative correlation with the number of McGurk responses ( $\mathrm{P}=0.001$, uncorrected, cluster size $\mathrm{Z} 20)$. See Table 1 for details of brain areas 1-7. (b) Mean con values (weighted effect sizes versus baseline) in brain areas 1-7 for the seven stimuli used. Error bars indicate standard errors. Stimuli: $1=$ AV $-510 \mathrm{~ms}, 2=$ AV $-110 \mathrm{~ms}, 3=$ AV50 ms, $4=$ AV210 ms, $5=$ AV410 $\mathrm{ms}, 6=\mathrm{A}, 7=\mathrm{V}$.

\section{Discussion}

\section{Audiovisual binding as a function of audiovisual delay}

The aim of this study was to correlate the brain activity patterns with a perceptual measure of AV binding as evoked by the McGurk effect. Our psychophysical experiment showed that four out of 20 individuals were insensitive to the McGurk effect. These individuals always perceived the auditory stimulus, /aba/ or /apa/, independently of AV synchronization, and apparently did not combine auditory and visual information in the identification task. Many other studies mention that typically $10-20 \%$ of individuals are insensitive to the McGurk effect (e.g. [6,7]) Gentilucci and Cattaneo [8] even report that over $50 \%$ of individuals are insensitive. Therefore, non-McGurk individuals form a substantial part of the normal population. In previous fMRI and PET studies that used the McGurk effect to study AV binding $[7,9,10]$, individuals were not screened to see whether they were sensitive to the McGurk effect or not, which might have resulted in contamination of these brain imaging data by including individuals who did not bind AV information. 
The psychophysical data as shown in Fig. 1 are comparable with those described in the literature [6,11], with prevalent AV fusion over temporal asynchronies from -30

to $+130 \mathrm{~ms}$. A window of AV integration of around $200 \mathrm{~ms}$ and with an asymmetrical prevalence for audio lags was also found in other studies, such as of a simultaneity judgment task [12] and in an electrophysiological study of the cat superior colliculus [13]. This asymmetric temporal window of integration could be caused by the difference in speed between mechanotransduction and phototransduction, acoustic mechanotransduction being around $60 \mathrm{~ms}$ faster than phototransduction [14]. Adaptation of the central nervous system to differences in the speed of light and sound resulting in an audio lag for sources at a distance from the perceiver has also been proposed to account for the facilitated AV integration of stimuli with leading visual stimulation [13].

\section{Brain regions with BOLD responses correlated to AV binding}

Our results show that the BOLD response is correlated to the strength of the McGurk effect in a select number of the brain areas: the supramarginal gyrus and superior frontal gyrus, thalamus and parts of the cerebellum. As the McGurk effect indicates AV binding, we hypothesize that these brain areas play a role in AV processing. Several other studies suggest that these brain areas are involved in AV or multisensory processing. The supramarginal gyrus are thought to be involved in AV speech perception $[15,16]$, and the supramarginal gyrus and superior frontal gyrus in AV motion perception [17], whereas several nuclei of the thalamus function as multimodal gateways of sensory information [18]. Further, the cerebellum is mentioned in many studies of AV processing and is thought to be activated during asynchrony detection [19] and AV speech perception [20], for instance.

\section{Decreased BOLD responses as a function of AV binding}

Additivity and super-additivity are used as criteria to indicate AV integration sites [21,22] in most brain imaging studies. We were, however, unable to find the brain areas in which the BOLD response positively correlated with the number of McGurk responses. On the other hand, our results are comparable with those of Jones and Callan [10], Miller and d'Esposito [16] and Bernstein et al. [15], who for example found in the supramarginal gyrus larger BOLD responses to temporally offset or incongruent stimuli than to synchronized or congruent AV stimulation. Bernstein hypothesized that in these brain areas temporally incongruent stimuli would give rise to a larger BOLD response because of the mismatch between auditory and visual information. However, as the monosensory A and V stimuli produce BOLD responses that are comparable with those produced by the offset AV stimuli in our study, it appears that the offset stimuli do not cause increased BOLD responses. Instead, the synchronous, integrated AV stimuli seem to produce decreased BOLD responses. Assuming that decreased BOLD responses represent decreased neuronal activity, this finding agrees with recent evidence based on single-cell recordings in cats, that activity is decreased in a substantial part of multisensory neurons in response to simultaneous multimodal stimulation [23]. The positive con values found after auditory-only, visual-only and AV stimulation can be explained by the assumption that these brain areas also contain neurons that are activated during AV or monosensory stimulation.

So far, we can only speculate on why we did not find a single brain area in which the BOLD response increased with AV binding. One difference between this study and many other studies of AV integration is that we used an active AV integration task, rather than a passive paradigm. There is evidence that attention demanding tasks can result in decreased brain activity levels in a network of brain areas [24,25]. However, as the task and probably also attention did not vary with the different stimuli used

in our study, the decreased BOLD response is probably not related to attention. Another important difference between our study and almost all other studies of AV integration is that we used a sparse sampling paradigm. Although we are convinced of the usefulness of sparse sampling because of the importance of a well-defined and easily discerned auditory stimulus, the price paid for using this 
paradigm is the reduction of statistical power and/or a longer duration for the scanning experiment.

Perhaps we were unable to identify relatively small positively-correlated BOLD responses because of the reduced statistical power of our experiment. However, it is clear that negativelycorrelated BOLD responses were much stronger than the possible positive correlations.

\section{Conclusion}

Two cortical brain areas, the supramarginal gyrus and the superior frontal gyrus, part of the thalamus and parts of the cerebellum showed activity patterns that correlated with the perception of the McGurk effect, used to indicate AV binding. BOLD responses decreased with increased AV binding in all these brain areas.

\section{Acknowledgements}

The authors wish to thank the subjects for participating and Professor Pim van Dijk and the anonymous reviewers for their valuable comments on an earlier draft of this manuscript.

\section{References}

1.McGurk H, MacDonald J. Hearing lips and seeing voices. Nature 1976; 264:746-748.

2.Miller GA, Nicely P. An analysis of perceptual confusions among some English consonants. J Acoust Soc Am 1955; 27:338-352.

3.Fisher CG. Confusions among visually perceived consonants. J Speech Hear Res 1968; 11:796-804.

4.Eden GF, Joseph JE, Brown HE, Brown CP, Zeffiro TA. Utilizing hemodynamic delay and dispersion to detect fMRI signal change without auditory interference: the behavior interleaved gradients technique. Magn Reson Med 1999; 41:13-20.

5.Hall DA, Haggard MP, Akeroyd MA, Palmer AR, Summerfield AQ, Elliott MR, et al. 'Sparse' temporal sampling in auditory fMRI. Hum Brain Mapp 1999; 7:213-223.

6.Munhall KG, Gribble P, Sacco L, Ward M. Temporal constraints on the McGurk effect. Percept Psychophys 1996; 58:351-362.

7.Sekiyama K, Kanno I, Miura S, Sugita Y. Auditory-visual speech perception examined by fMRI and PET. Neurosci Res 2003; 47:277-287.

8.Gentilucci M, Cattaneo L. Automatic audiovisual integration in speech perception. Experimental Brain Res 2005; 167:66-75.

9.Olson IR, Gatenby JC, Gore JC. A comparison of bound and unbound audio-visual information processing in the human cerebral cortex. Brain Res Cogn Brain Res 2002; 14:129-138.

10.Jones JA, Callan DE. Brain activity during audiovisual speech perception: an fMRI study of the McGurk effect. Neuroreport 2003; 14:1129-1133.

11.Wassenhove van V, Grant KW, Poeppel D. Temporal window of integration in auditory-visual speech perception. Neuropsychologia 2007; 45:598-607.

12.Stone JV, Hunkin NM, Porrill J, Wood R, Keeler V, Beanland M, et al. When is now? Perception of simultaneity. Proc R Soc Lond B Biol Sci 2001; 268:31-38.

13. Meredith MA, Nemitz JW, Stein BE. Determinants of multisensory integration in superior colliculus neurons. I. Temporal factors. J Neurosci 1987; 7:3215-3229.

14.Wallace MT, Stein BE. Development of multisensory neurons and multisensory integration in cat superior colliculus. J Neurosci 1997; 17:2429-2444.

15.Bernstein LE, Lu ZL, Jiang J. Quantified acoustic-optical speech signal incongruity identifies cortical sites of audiovisual speech processing. Brain Res 2008; 1242:172-184.

16.Miller LM, D'Esposito M. Perceptual fusion and stimulus coincidence in the cross-modal integration of speech. J Neurosci 2005; 25:5884-5893.

17. Baumann O, Greenlee MW. Neural correlates of coherent audiovisual motion perception. Cereb Cortex 2007; 17:1433-1443. 
18.Komura Y, Tamura R, Uwano T, Nishijo H, Ono T. Auditory thalamus integrates visual inputs into behavioral gains. Nat Neurosci 2005; 8:1203-1209.

19.Bushara KO, Grafman J, Hallett M. Neural correlates of auditory-visual stimulus onset asynchrony detection. J Neurosci 2001; 21:300-304.

20.Skipper JI, Nusbaum HC, Small SL. Listening to talking faces: motor cortical activation during speech perception. Neuroimage 2005; 25:76-89.

21.Calvert GA, Thesen T. Multisensory integration: methodological approaches and emerging principles in the human brain. J Physiol Paris 2004; 98:191-205.

22.Laurienti PJ, Perrault TJ, Stanford TR, Wallace MT, Stein BE. On the use of superadditivity as a metric for characterizing multisensory integration in functional neuroimaging studies. Exp Brain Res 2005; 166:289-297.

23. Meredith MA, Keniston LR, Dehner LR, Clemo HR. Crossmodal projections from somatosensory area SIV to the auditory field of the anterior ectosylvian sulcus (FAES) in Cat: further evidence for subthreshold forms of multisensory processing. Exp Brain Res 2006; 172:472-484.

24.McKiernan KA, Kaufman JN, Kucera-Thompson J, Binder JR. A parametric manipulation of factors affecting task-induced deactivation in functional neuroimaging. J Cogn Neurosci 2003; 15:394-408. 25.Shulman GL, Fiez JA, Corbetta M, Buckner RL, Miezin FM, Raichle ME, Petersens SE. Common blood flow changes across visual tasks: II. decreases in cerebral cortex. J Cogn Neurosci 1997; 9:648663. 